\title{
Study of Personalized Network Tutoring System Based on Emotional-cognitive Interaction
}

\author{
Manfei Qi ${ }^{1}$, Ding $\mathrm{Ma}^{2}$ and Wansen Wang ${ }^{1}$ \\ ${ }^{1}$ Information Engineering College, Capital Normal University, \\ Beijing, 100037, China \\ qimanfei@163.com,wansenw@126.com \\ ${ }^{2}$ Security and Protection Department, Chinese People's Public Security University, \\ Beijing, 102600, China \\ mading139@126.com
}

\begin{abstract}
Aiming at emotion deficiency in present Network tutoring system, a lot of negative effects is analyzed and corresponding countermeasures are proposed. The model of Personalized Network tutoring system based on Emotionalcognitive interaction is constructed in the paper. The key techniques of realizing the system such as constructing emotional model and adjusting teaching strategies are also introduced.
\end{abstract}

Keywords: Personalized Network tutoring system, affective model, cognitive model, teaching strategies

\section{Introduction}

Network tutoring system uses modern educational technologies to implement an ideal learning environment through integrating the information technology into curriculum, which can embody the learning styles of students' main-body function, reform the traditional teaching structure and the essence of education thoroughly [1].

Although the current Network tutoring system have many merits, many of them only treat the advanced information technology as the simple communication tools, and release some learning contents and exercise in the network [2]. This kind of movable textbook or electronic textbook is indifferent to the learners, which lacks of the interaction of emotion. Thus, some learning problems of the 
learners in the learning process can't be solved and perplexity of the psychology can't get help.

How to measure cognitive emotion of learners in the E-learning system and realize harmonious emotion interaction and adjust teaching strategies becomes an important research topic in the distance education [3] .In this paper, facial expression recognition is used to construct affective model and calculation of cognitive ability is used to construct cognitive model. Teaching strategies and learning behaviors are adjusted according to learners' emotion state and cognitive ability. Thus, the system based on Emotional- cognitive interaction could help the learners to pleasure study essentially.

\section{Personalized Network tutoring system model based on Emotional- cognitive interaction}

The model of Personalized Network tutoring system model based on Emotional- cognitive interaction is shown in Figure 1.

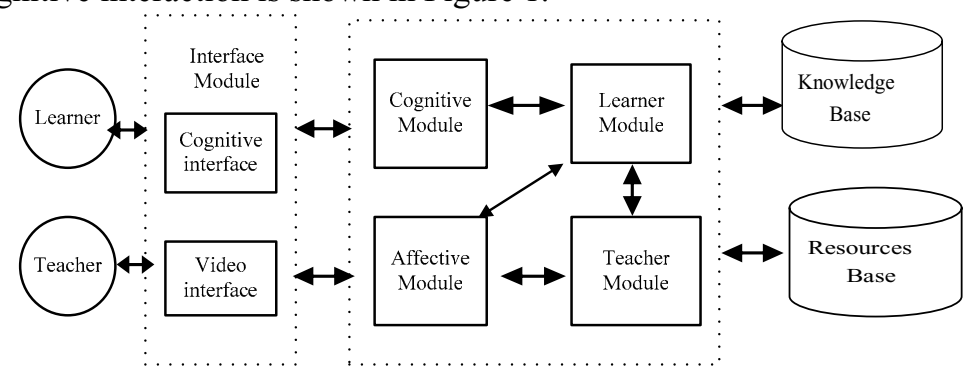

Fig. 1 the model of Personalized Network tutoring system based on Emotional- cognitive interaction

The model is composed of six modules as follows:

Interface module: It is interacting interface between Personalized Network tutoring system and learners and teachers.

Learner module: It manages all important information about the learners in the learning process. Such as student basic information, personal characteristics, historical behavior, learning aptitudes, test records etc.

Teacher module: It generates teaching strategies, which are adjusted according to the evaluation parameters of cognitive ability and learning records of learner module and provide learners appropriate teaching contents and teaching strategies. Simultaneously, emotional encouragement and compensation is also given.

Affective module: It analyses learner's facial expressions to show they are interested in learning or tired, happy or distressed. According to learners' facial expressions, with the learning mood of psychology research, access to learners 'psychological status, it is the basis for the adjustment of teaching strategies. 
Cognitive module: It is module response to the level of knowledge of the students. According to Bloom classification of the target will be divided cognitive ability into remember, understanding, application, analysis, synthesis and evaluation of six grades.

Knowledge base: It contains all characteristics of the knowledge to teach, storing information on the topics, tasks, excises, relationships between them, difficulty of each task, etc

\section{Implementation of key technologies}

\subsection{Construction of affective model}

There are many types of facial expressions, but emotional teaching, there is absolutely no need to study all the facial expressions, as long as they can seize those main emotion closely related to the learning process can be. The system analyzes the study expression from the following three aspects:

(1) Interest (that means the degree to elude), measured by the area of the face [4]. when the contour of the face detected becomes bigger compared with the normal state, it is suggested that learners are leaning forward during the learning process and are interested in the current subjects; in contrast, when the contour of the face detected becomes smaller, it is suggested that learners are leaning backward during the learning process and are not interested in the current subjects at all.

(2) Excitation (that is concentration), measured by eye spacing [4]. When the eye spacing becomes wider, it is suggested that learners are paying more attention to the current learning contents; in contrast, when the eye spacing becomes less narrow, it is suggested that learners are paying less attention to the current learning contents (tired emotionally). 


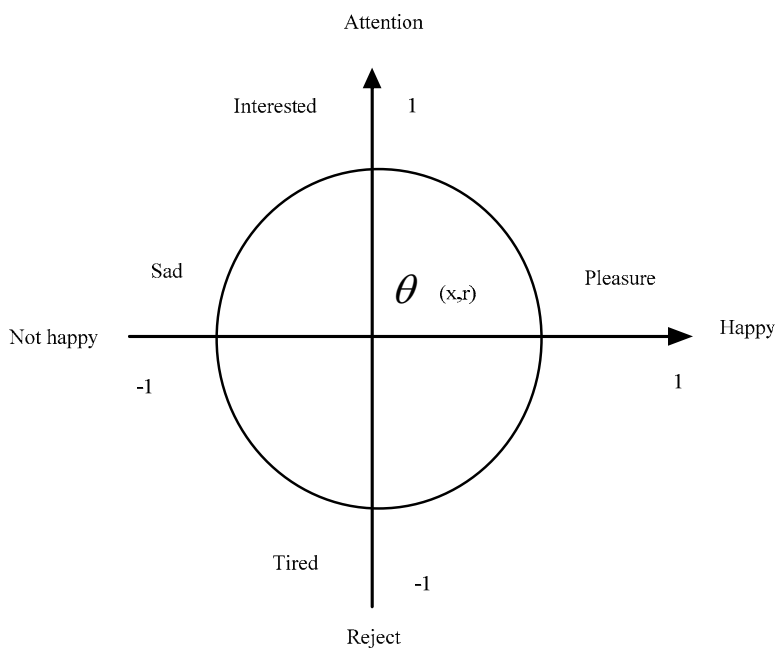

Fig.2 Two dimensional emotional spaces

(3) Happiness (roughly divided into pleasure and distress), measured by facial expressions. When facial expressions appear happy, it is suggested that learners are interested in learning stuff and can master them; when facial expressions appear distressed or confused, it is suggested that learners are not interested in learning stuff and can not master them at all.

According to the three groups of parameters above, we defined twodimensional emotion space, which are "happy- not happy" dimension and "attention- reject" dimension [5], as shown in figure 2. It contains four basic emotion states such as pleasure, sad, interested, and tired. All normal emotions are represented by a point located in a circle with the radius of 1.Points outside the circle stands for abnormal emotions and the origin stands for calm state. Pleasure and sad are a pair of opposite emotion, which stands for 1 and -1 along the horizontal axis, respectively; interested and tired are a pair of opposite emotion, which stands for 1 and -1 along the horizontal axis, respectively. which

$$
r=\sqrt{x^{2}+y^{2}} ; \theta=\left\{\begin{array}{l}
\arccos \left(x / \sqrt{x^{2}+y^{2}}\right), \text { if } y>0 \\
\pi+\arccos \left(x / \sqrt{x^{2}+y^{2}}\right), \text { if } y<0
\end{array}\right.
$$




\subsection{Calculation of cognitive ability}

Learners' cognitive ability scores of each knowledge point are stored in cognitive module. Values of all learners' six cognitive ability are defined as follows matrix.

$$
A=|R i j|
$$

Cognitive ability also depends on the time spent on learning knowledge point and the difficulty of knowledge point. Every knowledge point is assigned a standard time. Then, the coefficient of learning time can be got by comparing with the standard time. Assume $\mathrm{L}=\mathrm{S}$-time/U-time, where $\mathrm{S}$-time stands for standard time and $U$-time stands for actual learning time. Then, the learning time coefficient $T_{v}$ is defined as follows:

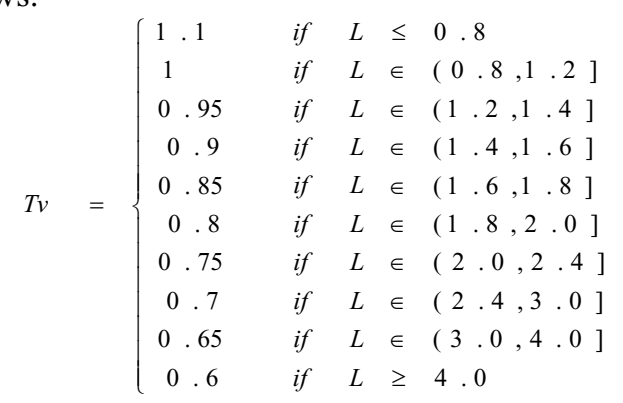

The coefficient between the difficulty of knowledge point and cognitive ability has the following form:

$$
D v=\left\{\begin{array}{lll}
1 & .2 & \begin{array}{l}
\text { difficult } \\
1
\end{array} \\
0 & .8 & \begin{array}{l}
\text { common } \\
\text { easy }
\end{array}
\end{array}\right.
$$

Based on all the factors above, the function to measure cognitive ability is:

$$
S=\left(\sum_{\substack{i=1 \\ j=1}}^{n, \sigma}(R i j \quad * V j \quad * T v \quad * D v \quad) / n\right.
$$

In the formula above, $V j$ are weight values of the six cognitive abilities, which are provided by experienced teachers. In this paper they are 0.1 for memory ability, 0.1175 for understanding ability, 0.1625 for application ability, 0.18 for analysis ability, 0.2225 for overall ability, and 0.2175 for evaluation ability [6]. Fuzzy cognitive ability will be grouped into five levels: (Lower, low, average, high, higher), lower, $\mathrm{s} \in[0,0.2)$;low, $\mathrm{s} \in[0.2,0.4)$; average , $\mathrm{s} \in[0.4,0.6)$; high, $\mathrm{s} \in[0.6,0.8)$; higher, $\mathrm{s} \in[0.8,1)$. 


\subsection{Emotional-cognitive interaction}

The relationship between emotion and cognition suggests that positive emotion promote learning and negative emotion prevents learning [7]. The change of learners' emotion is more complex in the learning process. If the learner has an idea to solve this problem, he will pleasure. If the learner is defeated repeatedly, he will suspect himself and changes into the sad state. If learners gaze at indifferent computer screens for a long time, they do not feel the interactive pleasure and understanding the problem, and they may have tired emotion. The system should apperceive this kind of emotional change and carry on emotion intervening, which make him turn to interested state and pay attention to study .Therefore, teaching strategies are adjusted dynamically according to the emotion and cognitive ability. Moreover, appropriate teaching strategies are generated by the inference engine to implement personalized learning. The system uses case-based reasoning (Figure 3).Figure 4 illustrates the process of reasoning in this system.

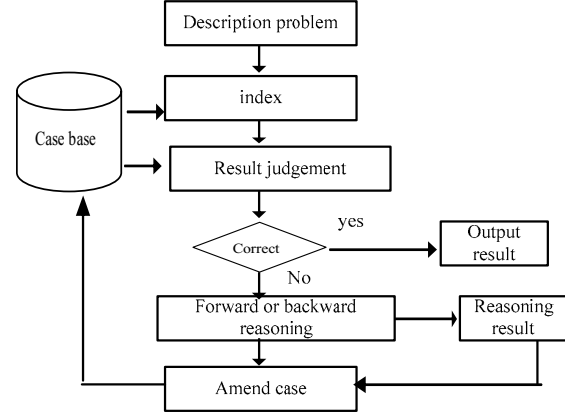

Fig.3 Case-based reasoning

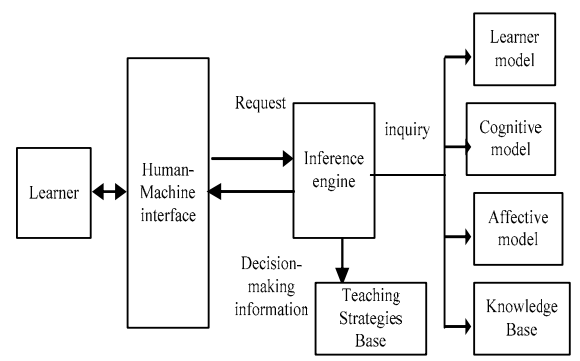

Fig.4 Process of reasoning

After learners enter the learning system, inference engine will inquire their learning history, testing records in the learner model, inquire knowledge point characteristics in the knowledge base, inquire their cognitive ability in cognitive model, and inquire the current emotion state in affective model. After that, the inference engine will compare the inquiry results and the rules in the teaching strategies base, if they are in good match, the operations described in the conclusions will be performed. Teaching strategies are adjusted through production rules:

IF $(<$ premise $1>,<$ premise $2>\ldots<$ premise $\mathrm{n}>$ )

THEN $(<$ Conclusion $1>$, $<$ Conclusion $2>\ldots<$ Conclusion $\mathrm{n}>$ )

For example:

IF $(<$ emotion state $=$ tired $>$ and $<$ cognitive ability $=$ average $>$ and $)$

THEN $(<$ Entering video learning $>)$

IF $(<$ emotion state $=$ sad $>$ and $<$ cognitive ability $=$ high $>)$

THEN $(<$ Entering dynamic interaction learning $>)$

In the learning process, the teaching strategies are not invariable. When learners' emotion state or cognitive ability changes, the system will analyze the reasons 
and will generate several teaching strategies. Then, it will choose the strategy that is most suitable for current emotion and knowledge point to learner.

\section{Conclusion}

This study proposes that Emotional-cognitive interaction is applied in the traditional Network tutoring system. The system based on Emotional-cognitive interaction may solve emotion deficiency in the great degree. The feedback information of facial expression and cognitive ability that is used in adjusting teaching strategies can pro-vide the personalized environment for learners. Based on it, emotion encouragement and compensation are also provided through the analysis of facial recognition. The re-sults obtained in this study demonstrate that emotion recognition in facial is feasible. It have broad prospect in modern education.

\section{Acknowledgements}

Project supported by Key science project from Beijing Municipal Commission of Education, that is nature science funded key project of Beijing (KZ200810028016)

\section{References}

[1] Kekang. He, "E-learning essence-information technology into curriculum". E-education Research, 2002,vol. 105, no. 1, .3-4.

[2] Jijun. Wang, "Emotion deficiency and compensation in distance learning" Chinese network edu-cation, 2005.

[3] Xirong. Ma, "Research on harmonious man-machine interaction model", Computer science, 2005

[4] Yinggang. Xie, "The Research of Intelligent E-Learning System Based on Artificial Psychology". University of science and Technology Beijing, 2007.

[5] Xiuyan. Meng etal, "Teaching assistant System Based on Affective Modeling", Application Re-search of Computer, 2007, Vol. 24, No. 4, 74-76.

[6] Weiying. Zheng, "Intelligent network tutoring on based personalized", FuDan university, 2006.

[7] Wansen. Wang etal, "Study of Intelligent Network tutoring System based on Artificial Emotion”, MINI-MICROSYSTEM, 2006, vol. 27, no. 3, 569-572 